\title{
Time-dependent Hartree-Fock-Bogoliubov calculations using a Lagrange mesh with the Gogny interaction
}

\author{
Yukio Hashimoto* \\ Division of Physics, Faculty of Pure and Applied Sciences, University of Tsukuba, Tsukuba 305-8571, Japan
}

(Received 19 July 2013; published 6 September 2013)

\begin{abstract}
A new numerical method of calculating the Hartree-Fock-Bogoliubov (HFB) and time-dependent HFB (TDHFB) with the Gogny interaction is proposed. The three-dimensional harmonic-oscillator (3DHO) basis functions are replaced by one-dimensional spatial grid points of Lagrange mesh plus two-dimensional harmonic-oscillator basis functions in the perpendicular plane to the direction of the spatial grid points [Lagrange mesh and harmonic oscillator (LMHO)]. By using the LMHO, the calculations of the HFB and TDHFB are carried out in the typical nuclei of ${ }^{20} \mathrm{O}$ and ${ }^{34} \mathrm{Mg}$ as an illustration of the feasibility of the TDHFB formulation with the LMHO. The strength functions of the quadrupole vibrations $(K=0)$ are obtained and are compared with the ones calculated with the 3DHO.
\end{abstract}

DOI: 10.1103/PhysRevC.88.034307

PACS number(s): 21.60.Jz, 21.30.Fe, 02.60.-x

\section{INTRODUCTION}

The nuclear mean-field theories have been widely used to investigate the static as well as the dynamical properties of nuclei. Among the mean-field methods, the time-dependent Hartree-Fock (TDHF) method has been playing the central role to investigate and to simulate the nuclear dynamical phenomena from the small-amplitude collective motions, such as giant resonances to the large-amplitude motions, such as fusion-fission processes [1-8].

Since the importance of the pairing correlations in the nuclei is well known, several groups have proposed the practical numerical methods of including the pairing effects to extend the TDHF to the time-dependent Hartree-Fock Bogoliubov (TDHFB) method [9-13]. In the numerical calculations of the TDHF and TDHFB, the Skyrme interactions [14] have mainly been used as the effective interactions in nuclei, and the wave functions are expressed on the spatial grid points (mesh). The spatial mesh is a useful basic tool to describe the particle continuum states properly, which is an advantage in the study of the dynamical role of the pairing correlations which couple the weakly bound states and positive energy states around the Fermi energy.

In the case of the Gogny interaction $[15,16]$, the practical cutoff of the energy range of the physical space is introduced naturally from the two-body interactions with the Gaussian functions. The harmonic-oscillator eigenfunctions are used as the basis functions to express the wave functions and matrix elements in the calculations with the Gogny interaction. The elongated nuclear shapes which appear in the fission process were described by adjusting the harmonic-oscillator parameter, e.g., $\omega_{z}$ and by introducing large spaces of basis functions [17]. A time-dependent method was proposed to describe the fissioning nucleus as well as the mass distributions in the fission fragments where the Hartree-Fock-Bogoliubov (HFB) solutions of the Gogny interaction with the constraints of the nuclear shapes were combined with the generator coordinate method [18].

\footnotetext{
*hashi@nucl.ph.tsukuba.ac.jp
}

The possibility of the mean-field calculation with the Gogny interaction by using the spatial grid points was proposed by Matsuse [19]. Taking account of the advantageous points of the spatial grid points in the study of the pairing correlations in the unstable nuclei, it would be worthwhile to extend Matsuse's idea of calculating the mean fields with the Gogny interaction on the spatial grid points to the realistic calculations of the Hartree-Fock (HF), HFB, TDHF, and TDHFB methods.

In this article, we propose a formulation which is an extension of the Gogny-TDHFB formulation with the basis functions of the three-dimensional harmonic-oscillator (3DHO) eigenfunctions [13]. In the new formulation, the basis functions along one of the three axes ( $z$ axis) are replaced by the spatial grid points, which make use of the Lagrange mesh (LM) [20]. This formulation is the first step toward the Gogny-TDHFB calculations on the three-dimensional spatial grid points with the aim at studying the dynamical role of the pairing correlations in the large-amplitude collective motions of the weakly bound nuclei. The formulation is expected to be applied to the other types of effective interactions with the finite ranges [21]. As the demonstration of the feasibility of the new formulation, we report the results of the HFB and TDHFB calculations of the linear responses of the typical nuclei by using the new formulation.

This article consists of the following sections: In Sec. II, the new formulation of the TDHFB with the LM is presented. In Sec. III, the results of the HFB calculations of the oxygen ${ }^{20} \mathrm{O}$ and magnesium ${ }^{34} \mathrm{Mg}$ are presented. In Sec. IV, the TDHFB with the LM is applied to the linear responses of the quadrupole $(K=0)$ vibrations in ${ }^{20} \mathrm{O}$ and ${ }^{34} \mathrm{Mg}$ in which the pairing correlations are active among the neutrons. Section $\mathrm{V}$ is for the summary and concluding remarks.

\section{TDHFB EQUATIONS WITH THE LAGRANGE MESH}

\section{A. Basic equations}

The nuclear Hamiltonian is assumed to take the form

$$
H=\sum_{\alpha \beta} T_{\alpha \beta} C_{\alpha}^{\dagger} C_{\beta}+\frac{1}{4} \sum_{\alpha \beta \gamma \delta} \mathcal{V}_{\alpha \beta \gamma \delta} C_{\alpha}^{\dagger} C_{\beta}^{\dagger} C_{\delta} C_{\gamma},
$$


where $T_{\alpha \beta}$ is the kinetic-energy matrix element and $\mathcal{V}_{\alpha \beta \gamma \delta}$ are antisymmetrized two-body matrix elements of the Gogny interaction. The operator $C_{\alpha}^{\dagger}\left(C_{\alpha}\right)$ is a nucleon creation (annihilation) operator of a state labeled with $\alpha$.

The Bogoliubov transformation from the particle operators $C_{\alpha}^{\dagger}$ and $C_{\alpha}$ into the quasiparticles $\beta_{k}^{\dagger}$ and $\beta_{k}$ is

$$
\begin{aligned}
& \beta_{k}^{\dagger}=\sum_{\alpha}\left(U_{\alpha k} C_{\alpha}^{\dagger}+V_{\alpha k} C_{\alpha}\right), \\
& \beta_{k}=\sum_{\alpha}\left(U_{\alpha k}^{*} C_{\alpha}+V_{\alpha k}^{*} C_{\alpha}^{\dagger}\right) .
\end{aligned}
$$

In the TDHFB method, we write the equations of motion for the matrices $U$ and $V$ in the Bogoliubov transformation (2) and (3) in the form [9]

$$
i \hbar \frac{\partial}{\partial t}\left(\begin{array}{l}
U(t) \\
V(t)
\end{array}\right)=\mathcal{H}\left(\begin{array}{l}
U(t) \\
V(t)
\end{array}\right),
$$

with the HFB Hamiltonian $\mathcal{H}$,

$$
\mathcal{H}=\left(\begin{array}{cc}
h & \Delta \\
-\Delta^{*} & -h^{*}
\end{array}\right) .
$$

The mean-field Hamiltonian $h$ and the pairing mean field $\Delta$ are introduced through the relations [14],

$$
\begin{aligned}
& h_{\alpha \beta}=T_{\alpha \beta}+\Gamma_{\alpha \beta}, \\
& \Gamma_{\alpha \beta}=\sum_{\gamma \delta} \mathcal{V}_{\alpha \gamma \beta \delta} \rho_{\delta \gamma}, \quad \Delta_{\alpha \beta}=\frac{1}{2} \sum \mathcal{V}_{\alpha \beta \gamma \delta} \kappa_{\gamma \delta},
\end{aligned}
$$

where $\rho$ and $\kappa$ are the normal density matrix and pairing tensor, respectively,

$$
\rho_{\alpha \beta}=\left(V^{*} V^{T}\right)_{\alpha \beta}, \quad \kappa_{\alpha \beta}=\left(V^{*} U^{T}\right)_{\alpha \beta} .
$$

\section{B. Matrix elements with the Lagrange mesh}

In Ref. [13], we used the $3 \mathrm{DHO}$ as the numerical basis functions. The basic idea in the present formulation is to replace the harmonic-oscillator eigenfunctions with the spatial grid points, i.e., the LM [20] in one of the directions ( $z$ axis) of the Cartesian coordinates.

In the present article, we make use of the LM with a set of spatial grid points which are located with an equal distance $s$. The basis functions $f_{l}(z)$ of the LM are [20]

$$
f_{l}(z)=\frac{1}{N} \frac{\sin \left[\pi\left(z-z_{l}\right) / s\right]}{\sin \left[\pi\left(z-z_{l}\right) / L\right]},
$$

with $L=N s$ and $N$ is the total number of the grid points on the $z$ axis. The basis functions $f_{l}(z)$ and the grid points $z_{l}$ are labeled by a set of index $l(l=1, \ldots, N)$. We have simple properties of the basis functions $f_{l}(z)$, such as $f_{k}\left(z_{k^{\prime}}\right)=\delta_{k k^{\prime}}$, the orthogonality relation [20],

$$
\int_{-L / 2}^{L / 2} f_{l}(z) f_{l^{\prime}}(z) d z=s \delta_{l l^{\prime}}
$$

and quadrature formula,

$$
\int_{-L / 2}^{L / 2} f_{l}(z) W(z) f_{l^{\prime}}(z) d z=s W\left(z_{l}\right) \delta_{l l^{\prime}}
$$

for a function $W(z)$.
In the calculation of the Gaussian part $V_{G}(\alpha \beta \gamma \delta)$ of the two-body matrix elements $\mathcal{V}_{\alpha \beta \gamma \delta}$,

$$
\begin{aligned}
V_{G}(\alpha \beta \gamma \delta)= & \iint \Phi_{\alpha}\left(\mathbf{r}_{1}\right)^{*} \Phi_{\beta}\left(\mathbf{r}_{2}\right)^{*} \exp \left(-\frac{1}{\mu^{2}}\left(\mathbf{r}_{1}-\mathbf{r}_{2}\right)^{2}\right) \\
& \times \Phi_{\gamma}\left(\mathbf{r}_{1}\right) \Phi_{\delta}\left(\mathbf{r}_{2}\right) d \mathbf{r}_{1} d \mathbf{r}_{2},
\end{aligned}
$$

with respect to the $3 \mathrm{DHO}$ basis functions $\Phi_{\alpha}(\mathbf{r})$,

$$
\Phi_{\alpha}(\mathbf{r})=\phi_{n_{x}(\alpha)}(x) \phi_{n_{y}(\alpha)}(y) \phi_{n_{z}(\alpha)}(z),
$$

we take into account the separable property of the Gaussian function with a range parameter $\mu$ into $x, y$, and $z$ directions,

$$
\begin{aligned}
& V_{G}(\alpha \beta \gamma \delta)= \prod_{k=x, y, z} V_{G}^{(k)}\left(n_{k}(\alpha), n_{k}(\beta), n_{k}(\gamma), n_{k}(\delta)\right), \\
& V_{G}^{(k)}\left(n_{k}(\alpha), n_{k}(\beta), n_{k}(\gamma), n_{k}(\delta)\right) \\
&=\iint \phi_{n_{k}(\alpha)}\left(r_{k 1}\right)^{*} \phi_{n_{k}(\beta)}\left(r_{k 2}\right)^{*} \\
& \quad \times \exp \left(-\frac{1}{\mu^{2}}\left(r_{k 1}-r_{k 2}\right)^{2}\right) \\
& \quad \times \phi_{n_{k}(\gamma)}\left(r_{k 1}\right) \phi_{n_{k}(\delta)}\left(r_{k 2}\right) d r_{k 1} d r_{k 2},
\end{aligned}
$$

where notations $r_{x} \equiv x, r_{y} \equiv y$, and $r_{z} \equiv z$ are used. The notations $\alpha, \beta, \gamma$, and $\delta$ are the labels of the 3DHO basis functions $\Phi_{\alpha}(\mathbf{r})$, which is a product of the one-dimensional harmonic-oscillator eigenfunctions $\phi_{n_{k}(\alpha)}(x)$. The notation $n_{k}(\alpha)$ denotes the number of quanta in the direction of $k(k=x, y, z)$ of the basis state $\alpha$. We have neglected the spin-isospin degrees of freedom in Eq. (14) for simplicity.

In the representation of the matrix elements where the basis functions $\left\{f_{l}(z) ; l=1, \ldots, N\right\}$ of the $\mathrm{LM}$ are introduced with respect to the direction of the $z$ axis, we replace the onedimensional harmonic-oscillator eigenfunction $\phi_{n_{z}(\alpha)}(z)$ with the basis function $f_{l}(z)$ and get a new set of the basis functions [Lagrange mesh and harmonic-oscillator (LMHO) basis],

$$
\Phi_{\alpha_{L}}^{L}(\mathbf{r})=\phi_{n_{x}\left(\alpha_{L}\right)}(x) \phi_{n_{y}\left(\alpha_{L}\right)}(y) f_{l\left(\alpha_{L}\right)}(z)
$$

where $\alpha_{L}$ is an index of the set of the labels $\left\{n_{x}\left(\alpha_{L}\right)\right.$, $\left.n_{y}\left(\alpha_{L}\right), l\left(\alpha_{L}\right)\right\}$. The indices $\beta_{L}, \gamma_{L}$, and $\delta_{L}$ are defined in a similar way.

Then, the matrix elements of the Gaussian part in Eq. (14) are changed into

$$
\begin{aligned}
V_{G L} & \left(\alpha_{L} \beta_{L} \gamma_{L} \delta_{L}\right) \\
= & \left\{\prod_{k=x, y} V_{G}^{(k)}\left(n_{k}\left(\alpha_{L}\right), n_{k}\left(\beta_{L}\right), n_{k}\left(\gamma_{L}\right), n_{k}\left(\delta_{L}\right)\right)\right\} \\
& \times V_{G}^{L}\left(l\left(\alpha_{L}\right), l\left(\beta_{L}\right), l\left(\gamma_{L}\right), l\left(\delta_{L}\right)\right),
\end{aligned}
$$

where the quantity $V_{G}^{L}\left(l\left(\alpha_{L}\right), l\left(\beta_{L}\right), l\left(\gamma_{L}\right), l\left(\delta_{L}\right)\right)$ is the matrix element of the Gaussian function in the direction of the $z$ axis,

$$
\begin{aligned}
V_{G}^{L} & \left(l\left(\alpha_{L}\right), l\left(\beta_{L}\right), l\left(\gamma_{L}\right), l\left(\delta_{L}\right)\right) \\
= & \iint f_{l\left(\alpha_{L}\right)}\left(z_{1}\right)^{*} f_{l\left(\beta_{L}\right)}\left(z_{2}\right)^{*} \exp \left(-\frac{1}{\mu^{2}}\left(z_{1}-z_{2}\right)^{2}\right) \\
& \times f_{l\left(\gamma_{L}\right)}\left(z_{1}\right) f_{l\left(\delta_{L}\right)}\left(z_{2}\right) d z_{1} d z_{2} \\
= & s^{2} \exp \left(-\frac{1}{\mu^{2}}\left(z_{l\left(\alpha_{L}\right)}-z_{l\left(\beta_{L}\right)}\right)^{2}\right) \delta_{l\left(\alpha_{L}\right), l\left(\gamma_{L}\right)} \delta_{l\left(\beta_{L}\right), l\left(\delta_{L}\right)} .
\end{aligned}
$$

In Eq. (18), the quadrature (11) is used. 
The matrix elements of the terms that include the $\delta$ function $\delta\left(\mathbf{r}_{1}-\mathbf{r}_{2}\right)$ instead of the Gaussian function are evaluated in a similar way as in the case of the Gaussian function.

In evaluating the matrix elements of the Coulomb potential $V_{C}\left(\mathbf{r}_{1}-\mathbf{r}_{2}\right)=\frac{1}{\left|\mathbf{r}_{1}-\mathbf{r}_{2}\right|}$, we followed the method given in Ref. [22] where $V_{C}\left(\mathbf{r}_{1}-\mathbf{r}_{2}\right)$ is transformed into an integral form

$$
\frac{1}{\left|\mathbf{r}_{1}-\mathbf{r}_{2}\right|}=\frac{2}{\sqrt{\pi}} \int_{0}^{\infty} \frac{d \mu}{\mu^{2}} \exp \left(-\frac{1}{\mu^{2}}\left(\mathbf{r}_{1}-\mathbf{r}_{2}\right)^{2}\right)
$$

with the Gaussian function $\exp \left(-\frac{1}{\mu^{2}}\left(\mathbf{r}_{1}-\mathbf{r}_{2}\right)^{2}\right)$. We make use of the matrix elements in (17) in calculating the matrix elements of the Coulomb part,

$$
\begin{aligned}
V_{C L}\left(\alpha_{L} \beta_{L} \gamma_{L} \delta_{L}\right)= & \iint \Phi_{\alpha_{L}}^{L}\left(\mathbf{r}_{1}\right)^{*} \Phi_{\beta_{L}}^{L}\left(\mathbf{r}_{2}\right)^{*} \frac{1}{\left|\mathbf{r}_{1}-\mathbf{r}_{2}\right|} \\
& \times \Phi_{\gamma_{L}}^{L}\left(\mathbf{r}_{1}\right) \Phi_{\delta_{L}}^{L}\left(\mathbf{r}_{2}\right) d \mathbf{r}_{1} d \mathbf{r}_{2} \\
= & \frac{2}{\sqrt{\pi}} \int_{0}^{\infty} \frac{d \mu}{\mu^{2}} V_{G L}\left(\alpha_{L} \beta_{L} \gamma_{L} \delta_{L} ; \mu\right),
\end{aligned}
$$

where the dependence of the matrix elements $V_{G L}\left(\alpha_{L} \beta_{L} \gamma_{L} \delta_{L} ; \mu\right)$ on the integral variable $\mu$ is explicitly expressed. By a variable transformation, the integral (20) with an infinite range of integration is changed into another integral form with a finite range of integration $[0,1]$. Then, the integral is calculated by applying the Gauss-Legendre quadrature formula [23].

\section{HFB equations}

By using the matrix elements with the LMHO in the previous subsection, we make the mean-field Hamiltonian $h_{\alpha_{L} \beta_{L}}$ and pairing mean field $\Delta_{\alpha_{L} \beta_{L}}$. The HFB equations [14],

$$
\left(\begin{array}{cc}
h-\lambda & \Delta \\
-\Delta^{*} & -h^{*}+\lambda
\end{array}\right)\left(\begin{array}{l}
U_{k} \\
V_{k}
\end{array}\right)=E_{k}\left(\begin{array}{l}
U_{k} \\
V_{k}
\end{array}\right)
$$

are solved to get the columns $U_{k}=\left(U_{\alpha_{L} k}\right)$ and $V_{k}=\left(V_{\alpha_{L} k}\right)$ together with the eigenvalues $E_{k}$ for the quasiparticle orbitals $k$. Here, $\lambda$ is the chemical potential to fix the number of protons or neutrons.

Starting from an initial guess of the set of the columns $U_{k}$ and $V_{k}$, i.e., the Bogoliubov transformation matrices $U$ and $V$, we follow the gradient method with the constraints of the particle numbers [14]. The total number of the quasiparticles which are taken into account as the physical space is restricted to be of the size in which the iterations of the gradient method in the HFB as well as the iterations of the time integration in the TDHFB are carried out within a practical CPU time.

\section{TDHFB equations}

By taking account of the unitarity property $w w^{\dagger}=w^{\dagger} w=$ 1 of the matrix $w=\left(\begin{array}{cc}U & V^{*} \\ V & U^{*}\end{array}\right)$ [14], we rewrite the equations of motion of the matrices $U$ and $V$ in Eq. (4),

$$
\begin{aligned}
i \hbar \frac{\partial}{\partial t} U_{\alpha k} & =\sum_{\beta}\left(h_{\alpha \beta} U_{\beta k}+\Delta_{\alpha \beta} V_{\beta k}\right), \\
i \hbar \frac{\partial}{\partial t} V_{\alpha k} & =-\sum_{\beta}\left(\Delta_{\alpha \beta}^{*} U_{\beta k}+h_{\alpha \beta}^{*} V_{\beta k}\right),
\end{aligned}
$$

into the following set of equations:

$$
\begin{aligned}
i \frac{\partial}{\partial t} U_{\alpha k} & =\sum_{l}\left\{U_{\alpha l} H_{11}(l k)-V_{\alpha l}^{*} H_{20}^{*}(l k)\right\}, \\
i \frac{\partial}{\partial t} V_{\alpha k}^{*} & =\sum_{l}\left\{U_{\alpha l} H_{20}(l k)-V_{\alpha l}^{*} H_{11}^{*}(l k)\right\},
\end{aligned}
$$

where $H_{11}$ and $H_{20}$ are defined as [14]

$$
\begin{aligned}
H_{11}(k l)= & \sum_{\alpha \beta} U_{\alpha k}^{*}\left(h_{\alpha \beta} U_{\beta l}+\Delta_{\alpha \beta} V_{\beta l}\right) \\
& -\sum_{\alpha \beta} V_{\alpha k}^{*}\left(h_{\alpha \beta}^{*} V_{\beta l}+\Delta_{\alpha \beta}^{*} U_{\beta l}\right), \\
H_{20}(k l)= & \sum_{\alpha \beta} U_{\alpha k}^{*}\left(h_{\alpha \beta} V_{\beta l}^{*}+\Delta_{\alpha \beta} U_{\beta l}^{*}\right) \\
& -\sum_{\alpha \beta} V_{\alpha k}^{*}\left(h_{\alpha \beta}^{*} U_{\beta l}^{*}+\Delta_{\alpha \beta}^{*} V_{\beta l}^{*}\right) .
\end{aligned}
$$

Here, the labels $\alpha$ and $\beta$ in Eqs. (24)-(27) are taken as the labels $\alpha_{L}$ and $\beta_{L}$ when the LMHO is used.

The integration of the set of Eqs. (24) and (25) is carried out in a similar way as used in the integration of Eqs. (22) and (23) [13]. The time is discretized into $t=n \Delta t(n=0,1,2, \ldots)$ with a time step $\Delta t$. We get the $(n+1)$ th solution from the $n$th one by the following relations:

$$
\left(\begin{array}{ll}
U & V^{*}
\end{array}\right)^{(n+1)}=\left(\begin{array}{ll}
U & V^{*}
\end{array}\right)^{(n)} \exp \left(-i \frac{\Delta t}{\hbar} \bar{H}_{\mathrm{HFB}}\right),
$$

where the intermediate Hamiltonian $\bar{H}_{\mathrm{HFB}}$ is given by

$$
\bar{H}_{\mathrm{HFB}}=\left(\begin{array}{cc}
\bar{H}_{11} & \bar{H}_{20} \\
-\bar{H}_{20}^{*} & -\bar{H}_{11}^{*}
\end{array}\right),
$$

and the matrices $\bar{H}_{11}$ and $\bar{H}_{20}$ are constructed by using Eqs. (26) and (27) in the procedure of the predictor-corrector method [13].

The former set of Eqs. (22) and (23) is equivalent to the latter [(24) and (25)] as long as the unitarity property $w w^{\dagger}=$ $w^{\dagger} w=1$ holds true. In the practical numerical calculations, however, we take a restricted number of quasiparticle orbitals $k$. We get more favorable results for the conservation of the energy and particle numbers by using the set of Eqs. (24) and (25) than the set of Eqs. (22) and (23) in the case of the TDHFB with the LMHO.

\section{HARTREE-FOCK-BOGOLIUBOV CALCULATIONS WITH THE LMHO}

The HFB formulation with the LMHO presented in the previous section was applied to ${ }^{20} \mathrm{O}$ in which the pairing correlation is active on the neutron side and the protons are in the normal state. The parameter set D1S of the Gogny interaction was used, and the parameters of the harmonicoscillator basis functions were $\hbar \omega_{x}=\hbar \omega_{y}=14.6 \mathrm{MeV}$.

In the calculations in this and the next section, the space of the harmonic-oscillator basis functions with respect to the $x$ and $y$ directions was taken to be $n_{x}+n_{y} \leqslant 4$. The space with respect to the $z$ axis was $-10 \mathrm{fm} \leqslant z \leqslant 10 \mathrm{fm}$. 


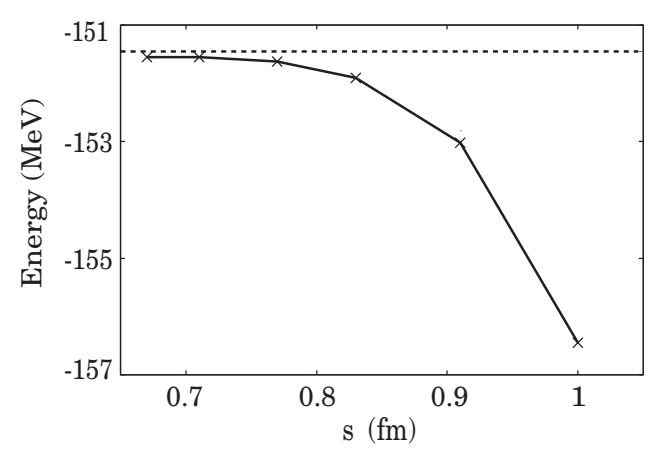

FIG. 1. HFB energy of oxygen ${ }^{20} \mathrm{O}$ with respect to mesh size $s$. The broken line is the HFB energy calculated with the 3DHO. Crosses $(\times)$ denote the mesh sizes $s=0.67,0.71,0.77,0.83,0.91$, and $1.0 \mathrm{fm}$ at which the HFB ground states are calculated.

The center-of-mass corrections were taken into account only in the mean-field Hamiltonian, and the pairing potentials were made from the Gaussian and $L S$ parts of the Gogny interaction.

Before starting the HFB iterations, we solved the BCS equations, which were expressed by using the $3 \mathrm{DHO}$ basis functions. The isotropic harmonic-oscillator parameters $\hbar \omega_{x}=\hbar \omega_{y}=\hbar \omega_{z}=14.6 \mathrm{MeV}$ were used. Then, the uv coefficients of the BCS solution were mapped on the Bogoliubov transformation matrices $U_{0}$ and $V_{0}$ expressed with the LMHO in (16). By starting from the matrices $U_{0}$ and $V_{0}$ as the initial trial matrices, the gradient method with the nucleon number constraints [14] was used to solve the HFB equations.

In Fig. 1, we show the total energies of the HFB solutions with respect to the mesh sizes $s=0.67,0.71,0.77,0.83,0.91$, and $1.0 \mathrm{fm}$. In the figure, the HFB energy changes smoothly when the mesh size $s$ decreases. The HFB energy with $s=$ $0.67 \mathrm{fm}$ is $-151.55 \mathrm{MeV}$, which is $0.11 \mathrm{MeV}$ lower than the HFB energy calculated with the 3DHO.

In Fig. 2, the variations in the energies of the components (kinetic-energy part, Gaussian mean-field part, $L S$ part, density-dependent part, Coulomb part, and Gaussian pairing part) in the total energy are shown with respect to the mesh size $s$. The variations in the energies are measured from the values at $s=1.0 \mathrm{fm}$.

From the left panel in Fig. 2, we see that the total energy is mainly determined by the kinetic energy, Gaussian mean-field energy, and density-dependent part energy. The dependence of the kinetic energy on the mesh size $s$ is rather moderate. Then, the tendency for the change in the density-dependent part energy is proportional to that for the Gaussian mean-field energy with an opposite sign: As the mesh size $s$ decreases from $s=1.0$ to $0.67 \mathrm{fm}$, the Gaussian part energy increases by $18 \mathrm{MeV}$, whereas, the energy of the density-dependent part decreases by $10 \mathrm{MeV}$. The changes in the kinetic energies are small compared with the Gaussian mean-field part and density-dependent part, and the decrease in the total kinetic energy is around $3 \mathrm{MeV}$ when the mesh size $s$ is changed from 1.0 to $0.67 \mathrm{fm}$. The change in the Gaussian pairing part is within $1 \mathrm{MeV}$, and the changes in the energies of the $L S$ part and Coulomb part are 0.5 and $0.1 \mathrm{MeV}$, respectively.
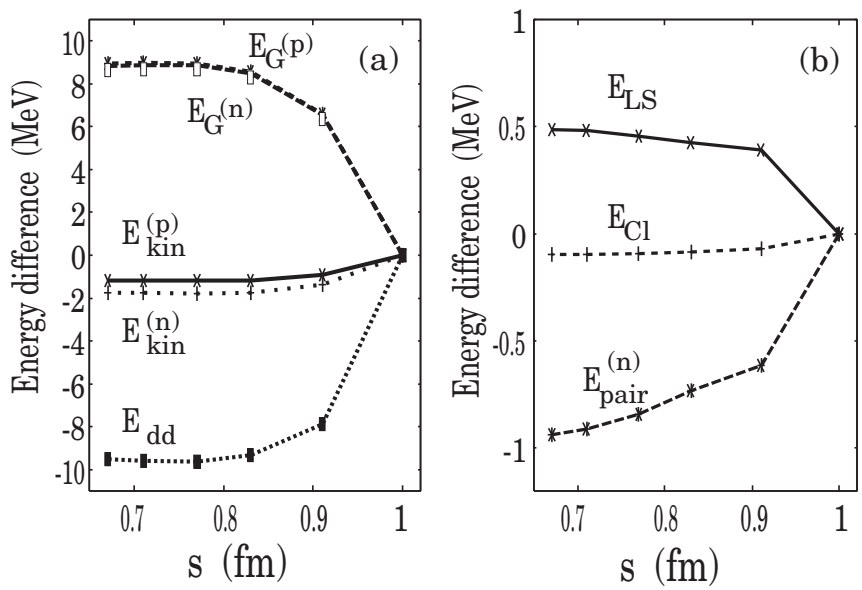

FIG. 2. Variations in the contributions of the components in the total energy of ${ }^{20} \mathrm{O}$ with respect to the mesh size $s$. The proton (neutron) kinetic energy $E_{\text {kin }}^{(p)}\left(E_{\text {kin }}^{(n)}\right)$, proton (neutron) Gaussian mean-field energy $E_{\mathrm{G}}^{(p)}\left(E_{\mathrm{G}}^{(n)}\right)$, density-dependent part energy $E_{\mathrm{dd}}, L S$ part energy $E_{L S}$, Coulomb mean-field energy $E_{\mathrm{Cl}}$, and neutron pairing energy $E_{\text {pair }}^{(n)}$ are displayed. Every quantity is measured from the value at $s=1.0 \mathrm{fm}$.

In Table I, several quantities (the HFB energy, neutron pairing energy, chemical potentials, and expectation values of the squared axis lengths) of the HFB ground state of ${ }^{20} \mathrm{O}$ calculated with the LMHO at $s=0.67 \mathrm{fm}$ are compared with those calculated with the $3 \mathrm{DHO}$. The difference between the HFB energy with the LMHO and the one with the $3 \mathrm{DHO}$ is $0.11 \mathrm{MeV}$. The magnitude of the neutron pairing energy is larger by $0.06 \mathrm{MeV}$ than the one with the 3DHO. The differences in the chemical potentials are within $0.01 \mathrm{MeV}$ on both the proton and the neutron sides. The shape of the density distribution with the LMHO is not exactly spherical but is slightly oblate with the deformation parameter $\beta=-0.0002$.

We took ${ }^{34} \mathrm{Mg}$ as an example of the ground state of the deformed nucleus with nonzero pairing energy. The parameters of the harmonic-oscillator basis functions with respect to the $x$ and $y$ axes were $\hbar \omega_{x}=\hbar \omega_{y}=12.55 \mathrm{MeV}$. The BCS solution was made by using the $3 \mathrm{DHO}$ with the parameters $\hbar \omega_{x}=$ $\hbar \omega_{y}=12.55$ and $\hbar \omega_{z}=9.65 \mathrm{MeV}$.

In Fig. 3, we show the total energies of the HFB solutions with respect to the mesh sizes $s=0.63,0.67,0.71,0.77,0.83$,

TABLE I. HFB ground-state quantities of ${ }^{20} \mathrm{O}$ calculated by using the LMHO and 3DHO. HFB energy $\left(E_{\text {total }}\right)$, neutron pairing energy $\left[E_{\text {pair }}(n)\right]$, chemical potentials $[\lambda(p)$ and $\lambda(n)]$, and expectation values of $x^{2}\left(\left\langle x^{2}\right\rangle\right), y^{2}\left(\left\langle y^{2}\right\rangle\right)$, and $z^{2}\left(\left\langle z^{2}\right\rangle\right)$ are displayed. The mesh size $s$ is $s=0.67 \mathrm{fm}$.

\begin{tabular}{lrr}
\hline \hline & LMHO & \multicolumn{1}{c}{ 3DHO } \\
\hline$E_{\text {total }}(\mathrm{MeV})$ & -151.56 & -151.45 \\
$E_{\text {pair }}(n)(\mathrm{MeV})$ & -6.84 & -6.78 \\
$\lambda(p)(\mathrm{MeV})$ & -15.24 & -15.23 \\
$\lambda(n)(\mathrm{MeV})$ & -5.35 & -5.35 \\
$\left\langle x^{2}\right\rangle\left(=\left\langle y^{2}\right\rangle\right)\left(\mathrm{fm}^{2}\right)$ & 53.22 & 53.18 \\
$\left\langle z^{2}\right\rangle\left(\mathrm{fm}^{2}\right)$ & 53.20 & 53.18 \\
\hline \hline
\end{tabular}




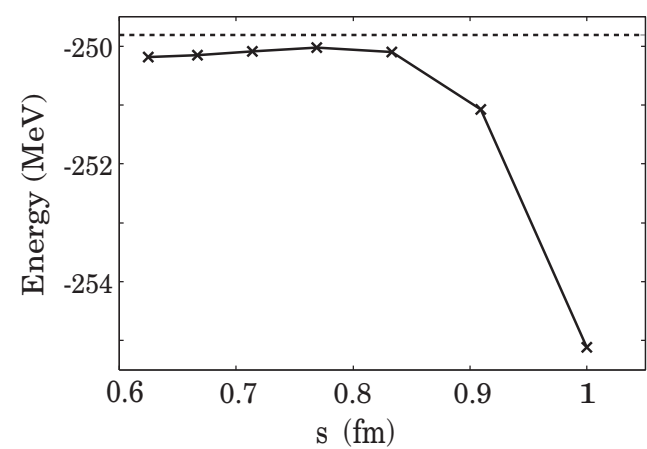

FIG. 3. HFB energy of magnesium ${ }^{34} \mathrm{Mg}$ with respect to mesh size $s$. The broken line is the HFB energy calculated with the 3DHO. Crosses $(\times)$ denote the mesh sizes $s=0.63,0.67,0.71,0.77,0.83$, 0.91 , and $1.0 \mathrm{fm}$ at which the HFB ground states are calculated.

0.91 , and $1.0 \mathrm{fm}$. When the mesh size $s$ is shifted to the smaller values, the HFB energy comes to a maximum value around $s=$ $0.8 \mathrm{fm}$. Then, the HFB energy changes smoothly with respect to the mesh size $s$, which is smaller than $0.77 \mathrm{fm}$. We note that there are cases of the mean-field calculations with the LM in which the dependence of the calculated mean-field energy on the mesh size is not necessarily monotonous but is like the damped oscillation about a converged value [24]. Since the dependence of the HFB energy on the mesh size is nonlinear and complicated, we need further studies to understand the convergence property with respect to the mesh size.

In Table II, the same quantities as in Table I are shown in the case with $s=0.67 \mathrm{fm}$. The HFB energy with the LMHO is deeper by $0.34 \mathrm{MeV}$ than that with the $3 \mathrm{DHO}$, and the magnitude of the neutron pairing energy is larger by $0.05 \mathrm{MeV}$. The proton chemical potential is deeper by $0.1 \mathrm{MeV}$ than the one with the 3DHO, whereas, the neutron chemical potentials coincide. The deformation of the density distribution with the LMHO is $\beta=0.435$, which is a little smaller than the value $\beta=0.438$ calculated with the $3 \mathrm{DHO}$.

\section{LINEAR-RESPONSE CALCULATIONS WITH THE LMHO}

We applied the TDHFB equations (24) and (25) with the LMHO to the quadrupole $(K=0)$ vibrations of ${ }^{20} \mathrm{O}$ and ${ }^{34} \mathrm{Mg}$ of which the properties of the HFB ground states were given in Sec. III. The parameters used in the TDHFB calculations were as follows: mesh size $s=0.91 \mathrm{fm}$, time step $c \Delta t=0.3 \mathrm{fm}$ with light speed $c$, and the number of lattice points $N=23$. The number of the quasiparticle orbitals was $k_{\max }=70$, which

TABLE II. The same as Table I but for ${ }^{34} \mathrm{Mg}$.

\begin{tabular}{lrr}
\hline \hline & LMHO & \multicolumn{1}{c}{ 3DHO } \\
\hline$E_{\text {total }}(\mathrm{MeV})$ & -250.15 & -249.81 \\
$E_{\text {pair }}(n)(\mathrm{MeV})$ & -4.65 & -4.60 \\
$\lambda(p)(\mathrm{MeV})$ & -19.88 & -19.78 \\
$\lambda(n)(\mathrm{MeV})$ & -2.67 & -2.67 \\
$\left\langle x^{2}\right\rangle\left(=\left\langle y^{2}\right\rangle\right)\left(\mathrm{fm}^{2}\right)$ & 99.33 & 99.06 \\
$\left\langle z^{2}\right\rangle\left(\mathrm{fm}^{2}\right)$ & 185.23 & 185.64 \\
\hline \hline
\end{tabular}
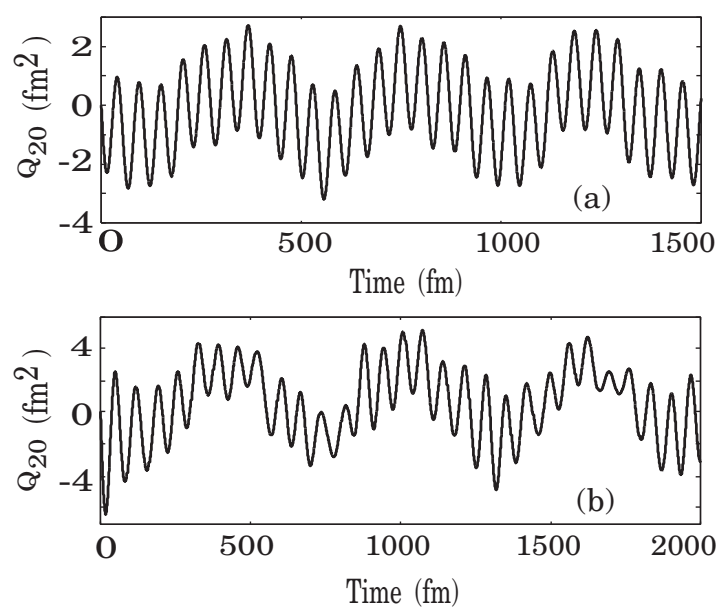

FIG. 4. Isoscalar quadrupole $(K=0)$ vibrations of (a) ${ }^{20} \mathrm{O}$ and (b) ${ }^{34} \mathrm{Mg}$ by using the TDHFB with the LMHO. In each of the panels, the values of $Q_{20}(t)$ are measured from the initial value $Q_{20}(t=0)$.

was just the number of the BCS orbitals used as the input of the HFB equations with the $3 \mathrm{DHO}$ in the space of the harmonic-oscillator quantum numbers $n_{x}+n_{y}+n_{z} \leqslant 4$. The initial condition was the impulse type with the strength parameter $\epsilon=0.001$ [13], which corresponds to the excitation energy of $20 \mathrm{keV}(76 \mathrm{keV})$ in ${ }^{20} \mathrm{O}\left({ }^{34} \mathrm{Mg}\right)$, respectively.

In Fig. 4, the variations in the quadrupole moment $Q_{20}$ with respect to the time are displayed. In the figure, the quadrupole moment $Q_{20}$ at every time step is measured from the initial value $Q_{20}(t=0)$ in each case of ${ }^{20} \mathrm{O}$ and ${ }^{34} \mathrm{Mg}$.

In the course of the time integration of the TDHFB equations, the deviation in the TDHFB energy $E_{\mathrm{TDHFB}}$ from the initial value $E_{0}$ in ${ }^{20} \mathrm{O}$ is displayed in panel (a) of Fig. 5. After integration up to $c t=1500 \mathrm{fm}$, the difference in the TDHFB energy from the initial value is on the order of $0.2 \mathrm{keV}$. Likely, the deviation in the expectation value of the neutron number $N_{\text {neut }}$ from the accurate value 12 with respect to the time is
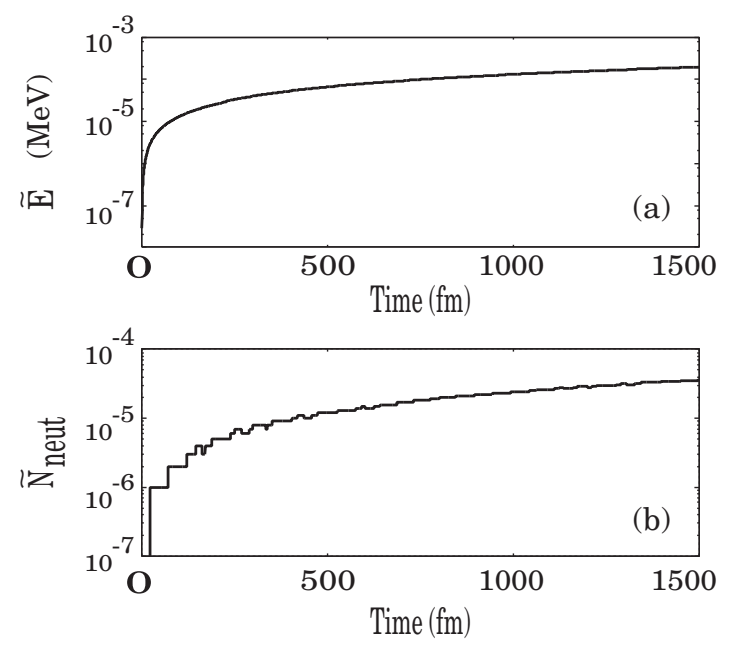

FIG. 5. (a) Deviation $\tilde{E}=E_{\mathrm{TDHFB}}-E_{0}$ of the TDHFB energy $E_{\mathrm{TDHFB}}$ from the initial value $E_{0}$ and (b) the neutron number $\tilde{N}_{\text {neut }}=$ $\left|N_{\text {neut }}-12\right|$ from the accurate value 12 with respect to the time in the isoscalar quadrupole vibrations of ${ }^{20} \mathrm{O}$ in Fig. 4. 

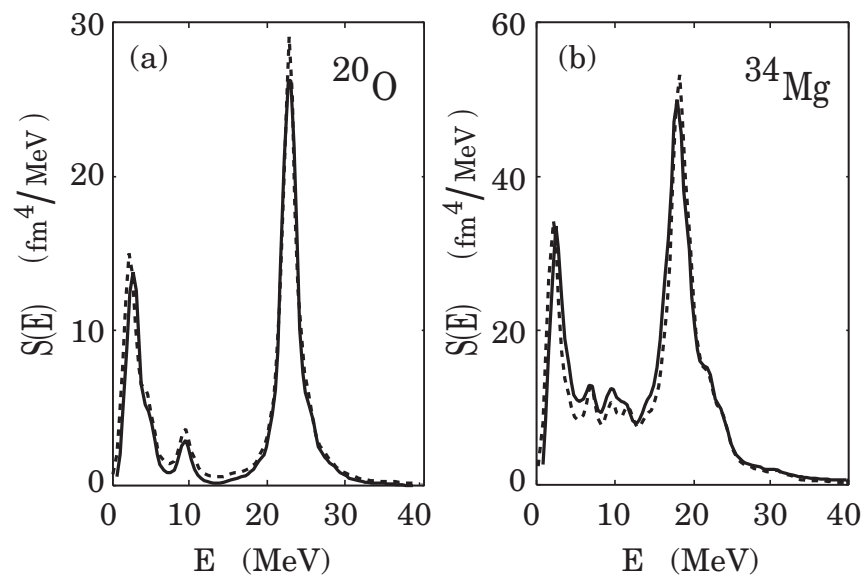

FIG. 6. Strength functions $S(E)$ of the isoscalar quadrupole $(K=0)$ vibrations of (a) ${ }^{20} \mathrm{O}$ and (b) ${ }^{34} \mathrm{Mg}$ in Fig. 4 with respect to the excitation energy $E$. The solid (broken) curves are the results with the LMHO (3DHO), respectively. Artificial widths are $1.0 \mathrm{MeV}$ in both panels (a) and (b).

displayed in panel (b) of Fig. 5. The expectation value of the neutron number is kept within the order of $10^{-5}$.

In the present calculations, the number of iterations of the predictor-corrector method in the course of the time integration is fixed at two. More accuracy in the conservation of the neutron number and the TDHFB energy is expected when the number of iterations of the predictor-corrector method is increased, although more CPU time is needed.

The strength functions of the quadrupole vibrations in Fig. 4 are shown in Fig. 6 together with the strength functions calculated with the $3 \mathrm{DHO}$.

In the case of ${ }^{20} \mathrm{O}$, the positions of the peaks of the strength function with the $\mathrm{LMHO}$ and those with the $3 \mathrm{DHO}$ are alike. We note that the position of the low-energy peak around $2.5 \mathrm{MeV}$ of the strength function with the LMHO is located on the larger energy side by $0.4 \mathrm{MeV}$ of the strength function with the 3DHO. Likely, in the case of ${ }^{34} \mathrm{Mg}$, the shape of the strength function with the LMHO is similar to the one with the 3DHO. The position of the low-energy peak around $2 \mathrm{MeV}$ of the strength function with the LMHO is $2.3 \mathrm{MeV}$, which corresponds to the one with the $3 \mathrm{DHO}$ at $1.9 \mathrm{MeV}$. The position of the high-energy peak around $18 \mathrm{MeV}$ of the strength function with the LMHO is $18.1 \mathrm{MeV}$, which corresponds to the one with the $3 \mathrm{DHO}$ at $18.3 \mathrm{MeV}$.

The detailed features of the strength functions are closely related with the ground-state properties of the nuclei. In order to obtain more accurate strength functions with the LMHO, we need further studies of the optimization of the parameters used in the LMHO, i.e., the harmonic-oscillator frequencies $\omega_{x}$ and $\omega_{y}$, the size $N_{\perp}$ of the space of the harmonic-oscillator quantum numbers $n_{x}+n_{y} \leqslant N_{\perp}$, the maximum number $k_{\max }$ of the quasiparticles, the mesh size $s$, and the total number of the lattice points $N$.

\section{SUMMARY AND CONCLUDING REMARKS}

In this article, we proposed a formulation in which the spatial grid points of the Lagrange mesh was introduced instead of the harmonic-oscillator basis functions with the aim at studying the role of the pairing correlations in the collective motions in the unstable nuclei with the Gogny interaction.

As the demonstration of the feasibility of the new formulation, we carried out: (1) the HFB calculations of the ground states of a spherical nucleus ${ }^{20} \mathrm{O}$ and an axially deformed nucleus ${ }^{34} \mathrm{Mg}$ and (2) the TDHFB calculations of the linear responses [quadrupole $(K=0)$ type] of ${ }^{20} \mathrm{O}$ and ${ }^{34} \mathrm{Mg}$.

The calculations were carried out under a restricted condition of the parameters: The spatial grids were set in the section of $z$ from -10 to $10 \mathrm{fm}$. The space of the harmonic-oscillator basis functions were $n_{x}+n_{y}+n_{z} \leqslant 4$, and the frequency parameters $\omega_{x}, \omega_{y}$, and $\omega_{z}$ were not the optimized ones.

We showed the dependence of the HFB ground-state energies on the mesh size $s$. The HFB energies were comparatively stable with respect to the change in the mesh size $s$ when it was smaller than $s \sim 0.8 \mathrm{fm}$. Among the components of the Gogny interaction, the changes in the energies of the Gaussian mean-field part and the density-dependent part were the largest in the section from $s=0.67$ to $1.0 \mathrm{fm}$ in ${ }^{20} \mathrm{O}$. The energies of the other components were rather moderate in the dependence on $s$. The chemical potentials and pairing energies in the HFB ground states with the LMHO were similar to those calculated with the $3 \mathrm{DHO}$ when the mesh size $s$ was smaller than $s \sim 0.8 \mathrm{fm}$.

In the TDHFB calculations, the deviations in the TDHFB energies from the initial values were around $1 \%$ of the initial excitation energies after 5000 steps of iterations. The deviations in the neutron number were on the order of $10^{-5}$. The shapes of the strength functions were similar to those calculated with the 3DHO as a whole, although there were some differences on a small scale. The optimization of the parameters, which were used in the present calculations, is necessary for us to study the detailed properties of the HFB ground states as well as the excited states through the TDHFB with the LMHO.

In the calculations of the HFB and TDHFB, a large part of the CPU time was used in making the matrix elements of the mean-field Hamiltonian by taking the traces of the twobody matrix elements with the density matrices and pairing tensors. The typical CPU time of one iteration in the TDHFB calculation of the linear response in ${ }^{34} \mathrm{Mg}$ (which includes one predictor loop plus two corrector loops) in the previous section was $170 \mathrm{~s}$ with 512 processors on a HITACHI SR16000M1.

The present formulation with the Gogny interaction in which the basis functions in the $z$ direction are the spatial grid points of the Lagrange mesh and the harmonic-oscillator basis functions are used in the $x$ and $y$ directions will be applied to the head-on collisions of the nuclei whose ground states are described by the HFB. Furthermore, the formulation will be extended into the one with the 3D spatial mesh. To realize the Gogny-TDHFB on the 3D spatial mesh and to apply it to the fusion-fission processes, it is essentially important to cut the CPU time. Improvements of the formulation and numerical methods into more efficient ones are now in progress.

\section{ACKNOWLEDGMENTS}

The author thanks Professor K. Matsuyanagi for discussions and encouragement. The author is also thankful 
to Professor J. Maruhn for discussions and comments. This research work was partly supported by the results of HPCI Systems Research Projects (Project

[1] P. Bonche, S. Koonin, and J. W. Negele, Phys. Rev. C 13, 1226 (1976).

[2] J. W. Negele, Rev. Mod. Phys. 54, 913 (1982).

[3] J. A. Maruhn, K. T. R. Davies, and M. R. Strayer, Phys. Rev. C 31, 1289 (1985).

[4] A. S. Umar, M. R. Strayer, R. Y. Cusson, P. G. Reinhard, and D. A. Bromley, Phys. Rev. C 32, 172 (1985).

[5] T. Nakatsukasa and K. Yabana, Phys. Rev. C 71, 024301 (2005).

[6] A. S. Umar and V. E. Oberacker, Phys. Rev. C 74, 021601(R) (2006).

[7] Y. Iwata, T. Otsuka, J. A. Maruhn, and N. Itagaki, Eur. Phys. J. A 42, 613 (2009).

[8] D. J. Kedziora and C. Simenel, Phys. Rev. C 81, 044613 (2010).

[9] Y. Hashimoto and K. Nodeki, arXiv:0707.3083.

[10] B. Avez, C. Simenel, and P. Chomaz, Phys. Rev. C 78, 044318 (2008).

[11] S. Ebata, T. Nakatsukasa, T. Inakura, K. Yoshida, Y. Hashimoto, and K. Yabana, Phys. Rev. C 82, 034306 (2010).
No. ID hp120204). Part of the numerical calculations was carried out on SR16000 at YITP at Kyoto University.
[12] I. Stetcu, A. Bulgac, P. Magierski, and K. J. Roche, Phys. Rev. C 84, 051309 (2011)

[13] Y. Hashimoto, Eur. Phys. J. A 48, 55 (2012).

[14] P. Ring and P. Schuck, The Nuclear Many-Body Problem (Springer-Verlag, Berlin, Heidelberg, 1980).

[15] D. Gogny, Nucl. Phys. A 237, 399 (1975).

[16] J. Dechargé and D. Gogny, Phys. Rev. C 21, 1568 (1980).

[17] W. Younes and D. Gogny, Phys. Rev. C 80, 054313 (2009).

[18] H. Goutte, J. F. Berger, P. Casoli, and D. Gogny, Phys. Rev. C 71, 024316 (2005).

[19] T. Matsuse, RIKEN Rev. 19, 18 (1998).

[20] D. Baye and P. Heenen, J. Phys. A 19, 2041 (1986).

[21] H. Nakada, Phys. Rev. C 68, 014316 (2003).

[22] M. Girod and B. Grammaticos, Phys. Rev. C 27, 2317 (1983).

[23] J. Dobaczewski et al., Comput. Phys. Commun. 180, 2361 (2009).

[24] H. Imagawa and Y. Hashimoto, Phys. Rev. C 67, 037302 (2003). 